Figure. Absolute change from baseline in $\mathrm{FVC}$ ( $\mathrm{mL}$ ) at week 52 in subjects in the SENSCIS trial versus hypothetical reference subjects without ILD matched for age, sex, ethnicity and height.

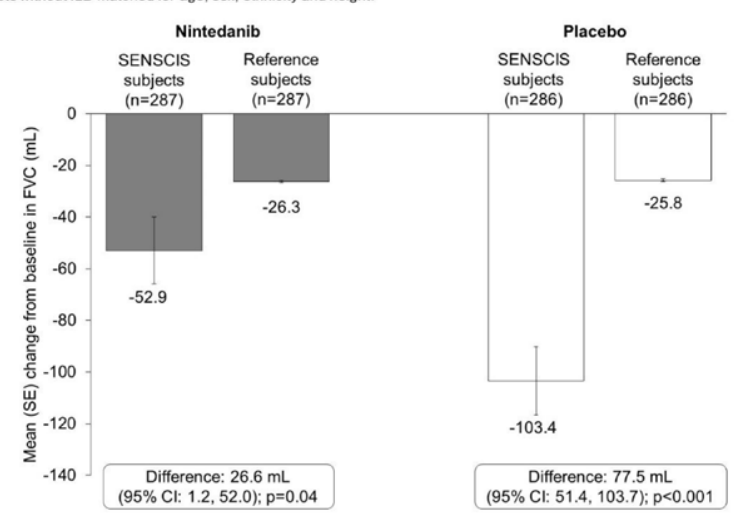

Acknowledgements: The SENSCIS trial was funded by Boehringer Ingelheim. Medical writing support was provided by FleishmanHillard Fishburn, London, UK The authors meet criteria for authorship as recommended by the International Committee of Medical Journal Editors (ICMJE).

Disclosure of Interests: Toby Maher Speakers bureau: Boehringer Ingelheim and Roche/Genentech, Consultant of: Acelleron Pharma, AstraZeneca, Boehringer Ingelheim, Bristol-Myers Squibb, GlaxoSmithKline and Roche/ Genentech, Arnaud Bourdin Speakers bureau: Actelion/Janssen (personal fees and other), AstraZeneca (personal fees and other), Boeringher Ingelheim (personal fees and other), Chiesi (personal fees and other), GlaxoSmithKline (personal fees and other), Novartis (personal fees and other), Pulsar Therapeutics (other), Roche (personal fees and other), Sanofi Regeneron (personal fees and other), Teva (other) and United Therapeutics (other), Consultant of: Actelion/ Janssen (personal fees and other), AstraZeneca (personal fees and other), Boeringher Ingelheim (personal fees and other), Chiesi (personal fees and other), GlaxoSmithKline (personal fees and other), Novartis (personal fees and other), Pulsar Therapeutics (other), Roche (personal fees and other), Sanofi Regeneron (personal fees and other), Teva (other) and United Therapeutics (other), Grant/research support from: Actelion/Janssen (grants and other), AstraZeneca (grants and other), Boeringher Ingelheim (grants and other), Chiesi (other), GlaxoSmithKline (grants and other), Novartis (other), Pulsar Therapeutics (other), Roche (other), Sanofi Regeneron (other), Teva (other) and United Therapeutics (other), Elizabeth Volkmann Consultant of: Boehringer Ingelheim, Grant/ research support from: Corbus and Forbius, Serena Vettori Paid instructor for: Boehringer Ingelheim, Consultant of: Boehringer Ingelheim, Jörg H.W. Distler Speakers bureau: Actelion, Active Biotech, AnaMar, Arxx Therapeutics, Bayer, Boehringer Ingelheim, Celgene, Galapagos NV, GlaxoSmithKline, Inventiva, JB Therapeutics, Medac, Pfizer, RuiYi and UCB, Consultant of: AnaMar, Arxx Therapeutics, Bayer, Boehringer Ingelheim, Galapagos NV, Inventiva, JB Therapeutics and UCB, Grant/research support from: Active Biotech, AnaMar, Array BioPharma, Arxx Therapeutics, aTyr, Bayer, Boehringer Ingelheim, Bristol-Myers Squibb, Celgene, Galapagos NV, GlaxoSmithKline, Inventiva, Novartis, SanofiAventis, Redx and UCB, Margarida Alves Employee of: Currently an employee of Boehringer Ingelheim, Christian Stock Employee of: Currently an employee of Boehringer Ingelheim, Oliver Distler Consultant of: AbbVie, Acceleron Pharma, Amgen, AnaMar, Arxx Therapeutics, Baecon Discovery, Bayer, Blade Therapeutics, Boehringer Ingelheim, ChemomAb, Corbus, CSL Behring, Galapagos NV, GlaxoSmithKline, Glenmark Pharmaceuticals, Horizon (Curzion) Pharmaceuticals, Inventiva, IQVIA, Italfarmaco, iQone, Kymera Therapeutics, Lilly, Medac, Medscape, Merck Sharp \& Dohme, Mitsubishi Tanabe Pharma, Novartis, Pfizer, Roche, Sanofi, Serodapharm, Target Bioscience, Topadur Pharma and UCB, Grant/research support from: Kymera Therapeutics and Mitsubishi Tanabe Pharma

DOI: 10.1136/annrheumdis-2021-eular.897

\section{POS0836 \\ CONDUCTION AND RHYTHM DISORDERS AMONG PATIENTS WITH SYSTEMIC SCLEROSIS: A US POPULATION BASED STUDY}

Y. Radwan ${ }^{1,2}$, R. Kurmann ${ }^{3}$, E. El-Am ${ }^{3}$, A. Sandhu' ${ }^{2}$, C. S. Crowson ${ }^{2,4}$, E. Matteson ${ }^{2}$, T. G. Osborn' ${ }^{2}$, K. J. Warrington' ${ }^{2}$, R. Mankad ${ }^{3}$, A. Makol $^{2}$. ${ }^{1}$ Michigan State University, Department of Internal Medicine, Lansing, United States of America; ${ }^{2}$ Mayo Clinic, Division of Rheumatology, Rochester, United States of America; ${ }^{3}$ Mayo Clinic, Department of Cardiovascular Disease, Rochester, United States of America; ${ }^{4}$ Mayo Clinic, Department of Health Sciences Research, Rochester, United States of America

Background: Systemic sclerosis (SSc) can impact multiple areas of the heart through fibrotic and vascular processes; leading to variable cardiac involvement including electrocardiogram (ECG) abnormalities. Conduction and rhythm disorders are associated with worse prognosis in patients with SSc. $(1,2)$

Objectives: To study the incidence, risk factors and outcomes of conduction and rhythm disorders in a US population-based cohort of patients with SSc and nonSSc comparators from the same geographic area.

Methods: A previously identified incident cohort of SSc patients (1980-2016) in a well-defined geographic area was compared to a randomly selected 2:1 cohort of age- and sex-matched non-SSc subjects from the same population base. Demographics, disease characteristics, cardiovascular risk factors and laboratory tests were abstracted by manual record review. ECGs and Holter ECGs were reviewed to determine the occurrence of any conduction or rhythm abnormalities. The need for cardiac interventions was also abstracted.

Results: 78 incident SSc cases and 156 non-SSc comparators were identified [age 56 years $\pm 15.7,91 \%$ female]. Prevalence of any conduction disorders before SSc diagnosis compared to non-SSc comparators was $15 \%$ vs. $7 \%(p=0.06)$ and any rhythm disorder was $18 \%$ vs. $13 \%(p=0.33)$. During a median follow up of 10.5 years in patients with SSc and 13.0 years in non-SSc comparators, conduction disorders developed in $25 \mathrm{SSc}$ patients with a cumulative incidence (ci) of $20.5 \%(95 \% \mathrm{Cl}: 12.4-34.1 \%)$ compared to 28 non-SSc patients with $\mathrm{cl}$ of $10.4 \%$ (95\% Cl: $6.2-17.4 \%$ ) (HR: $2.57 ; 95 \%$ Cl: $1.48-4.45)$, while rhythm disorders developed in $27 \mathrm{SSc}$ patients with ci of $27.3 \%$ (95\% Cl: $17.9-41.6 \%)$ vs 43 non-SSc patients with ci of $18.0 \%(95 \% \mathrm{Cl}: 12.3-26.4 \%)(\mathrm{HR}: 1.62 ; 95 \% \mathrm{Cl}$ : 1.00-2.64). (Figure 1).

Conduction disorders in patients with SSc during follow up included: 1st-degree atrioventricular block (AVB) $(n=12), 2 n d-$ degree AVB $(n=1)$, 3rd-degree AVB $(n=1)$, right bundle branch block $(n=10)$, left bundle branch block $(n=4)$, bifascicular block $(n=6)$, and prolonged-QT $(n=13)$. Rhythm disorders included: atrial fibrillation $(n=10)$, atrial flutter $(n=4)$, supraventricular tachycardia $(n=4)$, ventricular tachycardia $(n=1)$, and premature ventricular contractions $(n=16)$.

Pulmonary hypertension (PHT) was the only significant risk factor identified for development of both conduction and rhythm disorders ( $\mathrm{HR}=8.38,95 \% \mathrm{Cl}: 1.32$ 53.40 and $\mathrm{HR}=8.07,95 \% \mathrm{Cl}: 1.60-40.74$, respectively). Current smoking significantly increased the risk for development of rhythm disorders (HR=2.91, 95\% $\mathrm{Cl}$ 1.19-7.12). Conduction and rhythm disorders were associated with increased mortality among patients with SSc $(\mathrm{HR}=7.60,95 \% \mathrm{Cl}$ : $3.49-16.55$ and $\mathrm{HR}=4.87$, $95 \% \mathrm{Cl}: 2.28-10.42$, respectively, after adjusting for age, sex and calendar year of diagnosis).

Conclusion: Patients with SSc have a significantly higher prevalence of conduction disorders at disease onset than non-SSc comparators. During the course of their disease, their risk of developing conduction disorders is 2.6-fold, and risk of rhythm disorders is 1.6-fold increased, compared to non-SSc subjects.

PHT was significantly associated with increased risk of developing conduction and rhythm disorders among patients with SSc, a finding that should warran increased vigilance and screening for ECG abnormalities in this population.

\section{REFERENCES:}

[1] Tyndall A.J. et al. Ann Rheum Dis, 2010. 69(10): p. 1809-15.

[2] Desai C.S. et al. Curr Opin Rheumatol, 2011. 23(6): p. 545-54.
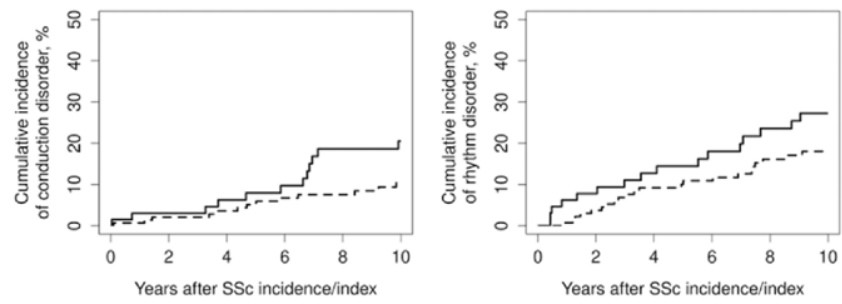

Figure 1. Cumulative incidence of any conduction or any rhythm disorder in SSc (solid line) vs non-SSc comparators (dashed line).

Disclosure of Interests: None declared DOI: 10.1136/annrheumdis-2021-eular.911

\section{\begin{tabular}{|l|l}
\hline POS0837 THE USE OF CANNABIDIOL IN THE TREATMENT OF \\
\hline
\end{tabular} PAIN RELATED TO SCLERODERMA DIGITAL ULCERS}

D. Giuggioli ${ }^{1}$, M. De Pinto ${ }^{1}$, L. Parenti ${ }^{1}$, L. Magnani ${ }^{2}$, P. Castrignanò ${ }^{1}$, C. Salvarani ${ }^{1,2}$, A. Spinella ${ }^{1} .{ }^{1}$ University of Modena and Reggio Emilia, Scleroderma Unit, Rheumatology Unit, Modena, Italy; ${ }^{2}$ AUSL-IRCCS of Reggio Emilia, Italy, Rheumatolgy, Reggio Emilia, Italy

Background: Systemic Sclerosis-SSc is an autoimmune disease, characterized by fibrosis due to immune-mediated microangiopathy. Digital ulcers-DUs represent frequent complications, they are recurrent, painful and often resistant to traditional treatments. Standard therapy, in particular oral opioids, is often 
inadequate or limited by side effect. Cannabidiol-CBD, is the major non-psychotropic component of the Cannabis sativa, recent studies on its effectiveness as an anxiolytic, anti-inflammatory, and antipsychotic drug showed promising results, in the setting of chronic pain too.

Objectives: We evaluated the efficacy of CBD drop in pain management in a cohort of SSc patients using standard rating scale VAS, PSQI and HAQ. We further assessed the safety profile and the potential use as opioid-sparing. Methods: From January to November 2019 we consecutively enrolled 31 SSc patients (F/M 26/5, mean age 53.0 $\pm 14.6 \mathrm{SD}$-years) referred to our Scleroderma Unit. All patients satisfied the EULAR/ACR SSc classification criteria. All cases were complicated by painful DUs resistant to analgesics and pain was classified as severe, according to WHO guidelines. CBD drops consist of cannabis sativa seed in olive oil, $10 \% \mathrm{CBD}$, laboratory tested to confirm a tetrahydrocannabinol-THC level $<0.3 \%$. The CBD oil was administrated sublingually twice-a-day. All patients started with CBD 3 drops twice-a-day, and progressively increased to the maximum dosage of 6 drops twice-a-day (from $27.6 \mathrm{~g}$ to $55.2 \mathrm{~g}$ dose/day). All patients continued local/systemic treatments for SSc: $24 / 31$ subjects performed calcium-channel blockers, 31/31 prostanoids infusion, 24/31 anti-endothelin drugs. All subjects were provided with a daily diary to record self-evaluation of pain using VAS, PSQI, hours of sleep per night, use of other analgesics, eventual side effects. HAQ-DI was also administrated. These indicators were assessed baseline and during follow-up. Safety of CBD was evaluated by patient's records of side effects. All data were analyzed by paired t-test. This investigation was a monocentric, prospective study. Ethical approval was obtained from the Competent Ethics Committee (protocol n. 282/15) and all participants gave written consent.

Results: CBD was administered for a mean period of $5.9 \pm 3.2 S D$-months. After the first month, VAS decreased from $94.80 \pm 8.72 S D$ to $54.70 \pm 9.40 S D$ $(p<0.0001)$, PSQI decreased from 9.27 $\pm 2.9 S D$ to $4.47 \pm 1.06 S D(p<0.001)$, total hours of sleep increased from $2.56 \pm 1.28$ SD to $5.67 \pm 0.85 S D(p<0.0001)$. The additional analgesic therapy was necessary in $22 / 31$ patients: $6 / 22$ only paracetamol, 12/22 paracetamol+oxycodone reducing the dosage of oxycodone at the minimum, $2 / 22$ oxycodone $20 \mathrm{mg}$ twice-a-day, 2/22 need fentanyl transdermal patch. After 3 months, VAS further reduced to $40.90 \pm 12.90$, PSQI decreased to $3.1 \pm 1.4 \mathrm{SD}$, the mean total hours of sleep per night was $6.10 \pm 0.79 \mathrm{SD}$ and the HAQ-DI decreased from 2.19 $\pm 0.67 \mathrm{SD}$ (baseline) to $0.79 \pm 0.46 \mathrm{SD}$ at the last patients' evaluation. At the end of the observation, 18/31 patients $(58 \%)$ showed DUs healing. We also interestingly reported improvement of dysphagia and appetite in $70 \%$, and an improvement in constipation related to opiods in $48 \%$. No patients experienced severe side effects in particular no psychoactive aspects. Mild side effects, namely dry mouth was referred by $9 / 31(29 \%)$, mild abdominal pain and changes in appetite by $10 / 31$ (32\%). No interaction with other drugs was observed.

Conclusion: Our study suggests that oral CBD is effective and safe in maintaining analgesia in SSc patients with DUs. Furthermore, CBD could be helpful in opioids tapering and to treat dysphagia, even if these observations need focused investigations. In conclusion, CBD might be a useful tool to manage chronic pain in SSc-DUs. These data provide a compelling rationale for further research to clarify the therapeutic potential of CBD in SSc.

Disclosure of Interests: None declared

DOI: 10.1136/annrheumdis-2021-eular.918

\section{POS0838 1 EPIDEMIOLOGY AND TRENDS IN SURVIVAL OF SYSTEMIC SCLEROSIS IN OLMSTED COUNTY: A POPULATION-BASED STUDY (1980-2018)}

C. Coffey ${ }^{1}$, Y. Radwan ${ }^{2}$, A. Sandhu ${ }^{3}$, C. S. Crowson ${ }^{1,4}$, P. Bauer ${ }^{5}$, E. Matteson ${ }^{1,4}$, A. Makol ${ }^{1}$. ${ }^{1}$ Mayo Clinic, Division of Rheumatology, Rochester, MN, United States of America; ${ }^{2}$ Michigan State University, Internal Medicine, Lansing, MI, United States of America; ${ }^{3}$ Kettering Health, Internal Medicine, Kettering, $\mathrm{OH}$, United States of America; ${ }^{4}$ Mayo Clinic, Health Sciences Research, Rochester, MN, United States of America; ${ }^{5}$ Mayo Clinic, Division of Pulmonary and Critical Care, Rochester, MN, United States of America

Background: Systemic sclerosis (SSc) is a complex immune-mediated disease with heterogeneous manifestations, which is characterized by vasculopathy and fibrosis of the skin and visceral organs. Mortality associated with SSc exceeds that of other rheumatic diseases, though population-based studies assessing recent trends in survival are lacking.

Objectives: We aimed to determine the incidence and prevalence of physician-diagnosed SSc in a population-based cohort over a 39-year time period, and assess for trends in survival over time.

Methods: Medical records of patients with a diagnosis or suspicion of SSc in a geographically well-defined area from Jan 1, 1980 to Dec 31, 2018 were reviewed to identify incident cases of SSc. Cases were defined by physician diagnosis of SSc, and fulfillment of the 2013 ACR/EULAR classification criteria was ascertained. Prevalent cases of SSc on Jan 1, 2015 were also identified. Incidence and prevalence rates were age- and sex-adjusted to the 2010 U.S. white population. Survival rates were compared with expected rates in the general population.

Results: 85 incident cases of SSc (91\% female, mean age $55.4 \pm 16$ y) and 49 prevalent cases on Jan 1, 2015 were identified. Patients had a mean 11.7 (SD 9.4) years of follow-up available. The overall age and sex adjusted annua incidence for $1980-2018$ was 2.5 (95\% Cl: $2.0-3.1)$ per 100,000 population, with no change in incidence over time $(p=0.32)$. The age-adjusted incidence was 4.4 (95\% Cl: 3.4-5.4) for females, and 0.56 (95\% Cl: $0.16-0.96)$ for males per 100,000 population. The age- and sex-adjusted prevalence on Jan 1, 2015 was 43.6 (95\% Cl: 31.3-55.8) per 100,000 population.

77 (91\%) patients fulfilled the 2013 classification criteria; 38 (45\%) fulfilled 1980 criteria. $70(82 \%)$ had limited cutaneous involvement, $12(14 \%)$ had diffuse cutaneous involvement, and $3(4 \%)$ had sine scleroderma. At SSc diagnosis, $80(94 \%)$ patients had Raynaud's, 43 (51\%) had sclerodactyly, 39 (46\%) had telangiectasias, 14/48 (29\%) had abnormal nailfold capillaries, 16/35 (46\%) had digital ulcers or fingertip scarring, 8 (9\%) had interstitial lung disease (ILD), and 7 (8\%) had pulmonary arterial hypertension (PAH). 77/82 patients (91\%) had a positive antinuclear antibody. $44(52 \%)$ had a known SSc-related autoantibody: 32 (73\%) with anti-centromere, 9 (20\%) with anti-Scl-70, and 4 (9\%) with anti-RNA-polymerase III.

Survival was $77 \%(95 \% \mathrm{Cl}: 69-87)$ at 5 years, $66 \%(95 \% \mathrm{Cl}: 56-78)$ at 10 years, and $42 \%(95 \% \mathrm{Cl}: 30-57)$ at 20 years, with no evidence of improved survival over time $(p=0.46)$. Age (Hazard ratio [HR]: 1.49 per 10 year increase; $95 \% \mathrm{Cl}$ 1.19-1.88), smoking at time of diagnosis (HR: $2.37 ; 95 \% \mathrm{Cl}: 1.05-5.34$ ), digital ischemia (HR: 2.54; 95\% Cl: 1.33-4.87), ILD (HR: 4.00; 95\% Cl: 2.11-7.59), and PAH (HR: 4.30; 95\% Cl: 2.24-8.25) had significant associations with mortality Survival of patients with SSc was poorer than the general population (standardized mortality ratio: $2.48 ; 95 \% \mathrm{Cl}: 1.76-3.39)$.

Conclusion: The average incidence of SSc in this population-based cohort spanning 39 years was 2.5 per 100,000 population, with no change in incidence over time. Age, smoking, digital ischemia, ILD and PAH were risk factors fo poorer survival. Overall survival for patients with SSc is worse than that of the general population and shows no improvement over time, suggesting continued need for improved diagnostic and treatment measures.

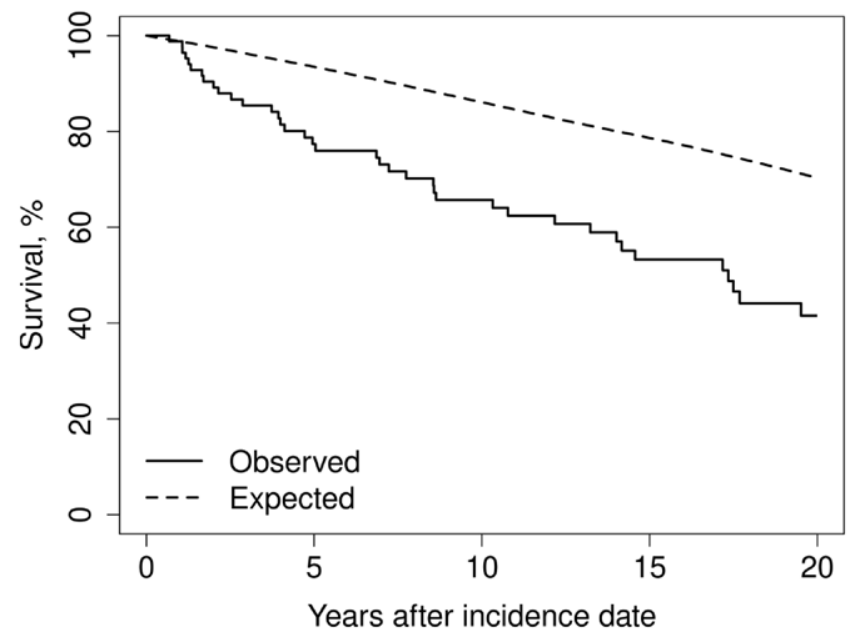

Figure 1. Survival of 85 Olmsted County residents with SSc compared with expected survival rates from Minnesota lifetables (observed: solid line, expected: dashed line).

Disclosure of Interests: None declared

DOI: 10.1136/annrheumdis-2021-eular.919

\section{POS0839 BODY COMPOSITION AND FREQUENCY OF SARCOPENIA IN PATIENTS WITH SYSTEMIC SCLEROSIS}

A. Efremova $^{1}$, O. Nikitinskaya ${ }^{1}$, N. Toroptsova ${ }^{1}$, O. Dobrovolskaya ${ }^{1}$, N. Demin ${ }^{1}$

${ }^{1}$ V.A. Nasonova Research Institute of Rheumatology, Department of Osteoporosis, Moscow, Russian Federation

Background: Sarcopenia can be age associated (primary form) or secondary to chronic disorders, including rheumatic inflammatory disorders. Systemic sclerosis (SSc) is a chronic autoimmune rheumatic disease characterized by widespread vasculopathy, progressive fibrosis of the skin and other internal organs, such as lung, kidneys, gastrointestinal tract, cardiovascular system. Different from the other chronic rheumatic inflammatory disorders, sarcopenia has not been well evaluated in SSc patients. 